\title{
Effect of hemoadsorption during cardiopulmonary bypass surgery - a blinded, randomized, controlled pilot study using a novel adsorbent
}

Martin H. Bernardi ${ }^{1 *}$, Harald Rinoesl ${ }^{1}$, Klaus Dragosits ${ }^{2}$, Robin Ristl ${ }^{3}$, Friedrich Hoffelner ${ }^{4}$, Philipp Opfermann ${ }^{1}$, Christian Lamm², Falk Preißing ${ }^{2}$, Dominik Wiedemann ${ }^{4}$, Michael J. Hiesmayr ${ }^{1}$ and Andreas Spittler ${ }^{2,5}$

\begin{abstract}
Background: Cardiopulmonary bypass (CPB) surgery initiates a systemic inflammatory response, which is associated with postoperative morbidity and mortality. Hemoadsorption (HA) of cytokines may suppress inflammatory responses and improve outcomes. We tested a new sorbent used for HA (CytoSorb ${ }^{\mathrm{TM}}$; CytoSorbents Europe $\mathrm{GmbH}$, Berlin, Germany) installed in the CPB circuit on changes of pro- and anti-inflammatory cytokines levels, inflammation markers, and differences in patients' perioperative course.
\end{abstract}

Methods: In this first pilot trial, 37 blinded patients were undergoing elective CPB surgery at the Medical University of Vienna and were randomly assigned to HA $(n=19)$ or control group $(n=18)$. The primary outcome was differences of cytokine levels (IL-1 $\beta$, IL-6, IL-18, TNF-a, and IL-10) within the first five postoperative days. We also analyzed whether we can observe any differences in ex vivo lipopolysaccharide (LPS)-induced TNF-a production, a reduction of high-mobility box group 1 (HMGB1), or other inflammatory markers. Additionally, measurements for fluid components, blood products, catecholamine treatment, bioelectrical impedance analysis (BIA), and 30-day mortality were analyzed.

Results: We did not find differences in our primary outcome immediately following the HA treatment, although we observed differences for IL-10 24 hours after CPB (HA: median 0.3, interquartile range (IQR) 0-4.5; control: not traceable, $P=0.0347$ ) and 48 hours after CPB (median 0, IQR $0-1.2$ versus not traceable, $P=0.0185$ ). We did not find any differences for IL-6 between both groups, and other cytokines were rarely expressed. We found differences in pretreatment levels of HMGB1 (HA: median 0, IQR 0-28.1; control: median 48.6, IQR 12.7-597.3, $P=0.02083$ ) but no significant changes to post-treatment levels. No differences in inflammatory markers, fluid administration, blood substitution, catecholamines, BIA, or 30-day mortality were found.

Conclusions: We did not find any reduction of the pro-inflammatory response in our patients and therefore no changes in their perioperative course. However, IL-10 showed a longer-lasting anti-inflammatory effect. The clinical impact of prolonged IL-10 needs further evaluation. We also observed strong inter-individual differences in cytokine levels; therefore, patients with an exaggerated inflammatory response to CPB need to be identified. The implementation of HA during CPB was feasible.

Trial registration: ClinicalTrials.gov: NCT01879176, registration date: June 7, 2013.

Keywords: Cytokines, Cytokine storm, CytoSorb, Cardiac surgery, Cardiopulmonary bypass, Hemadsorption, Inflammation, Interleukin, High-mobility box group 1

\footnotetext{
* Correspondence: martin.bernardi@meduniwien.ac.at

${ }^{1}$ Department of Cardiothoracic and Vascular Anaesthesia and Intensive Care

Medicine, Medical University of Vienna, Waehringer Guertel 18-20, A-1090

Vienna, Austria

Full list of author information is available at the end of the article
} 


\section{Background}

Cardiopulmonary bypass (CPB) surgery initiates a systemic inflammatory response induced by extrinsic and intrinsic factors [1-3]. Monocytes and high-mobility group box 1 protein (HMGB1), a chromatin protein, encoded by the Hmgbl gene in humans, are important players in systemic inflammation and belong to the main producers of pro- and anti-inflammatory cytokines $[4,5]$. Once activated by the extracorporeal circuit, they might lead to a dysregulation of inflammatory homeostasis and increased levels of both, pro- and anti-inflammatory plasma mediators such as tumor necrosis factor-alpha (TNF- $\alpha$ ), interleukin-1 $\beta$ (IL-1 $\beta$ ), IL-6, IL-10, and IL-18 [4, 6-9]. This strong inflammatory response induces post-surgical monocyte immunosuppression which is indicated by an impaired production of ex vivo lipopolysaccharide (LPS)induced TNF- $\alpha$ exaggeration [10].

All of these factors may lead to a prolonged postoperative course, including a delayed weaning from mechanical ventilation, recovery of organ functions, and discharge from the intensive care unit (ICU). Thus, measures to decrease the inflammatory process have the potential to improve the perioperative course [11]. Hemoadsorption (HA) using the CytoSorb ${ }^{\text {тм }}$ adsorber (CytoSorbents Europe $\mathrm{GmbH}$, Berlin, Germany) is a recent technology that has shown rapid elimination of many key cytokines that cannot be filtered by using current blood purification techniques [12].

The primary aim of this first single-center, blinded, randomized, and controlled pilot study was to investigate differences of pro- and anti-inflammatory cytokines in patients undergoing cardiac surgery with $\mathrm{CPB}$ using the CytoSorb ${ }^{\mathrm{TM}}$ adsorber compared with a control group within the first 5 postoperative days (POD). Furthermore, we investigated whether we can observe any differences in ex vivo LPS-induced TNF- $\alpha$ production, a reduction of HMGB1, or other inflammatory markers. Also, we investigated differences in fluid management or the use of catecholamines and differences in edema formation as determined by analysis of body composition by bioelectrical impedance analysis (BIA). Additionally, we compared length of ICU stay, respirator therapy, and 30-day mortality.

\section{Methods}

\section{Ethics approval}

This study was approved by the ethics committee of the Medical University of Vienna with reference number EK Nr: 1095/2013. Furthermore, we reported the study to the Austrian Federal Office for Safety in Health Care (INS-621000-0505) and registered it at ClinicalTrials.gov (NCT01879176) before recruitment started. Written informed consent to participate and consent to publish were obtained from each patient.

\section{Study design and patients}

This study was a randomized, blinded (in patients), controlled, single-center trial in 46 adult patients undergoing elective open heart surgery (coronary artery bypass graft [CABG], valve surgery, combined procedure) with an expected CPB duration of more than 120 minutes at the Department of Cardiac Surgery, Medical University of Vienna, Vienna, Austria. The study was conducted between Sept. 10, 2013, and May 6, 2015, at our department.

We excluded the following interventions or conditions: declined informed consent, transplant surgery, scheduled insertion of a cardiac assist device, thrombendarterectomy of the pulmonary arteries, emergency and urgent procedures, serum creatinine of more than $2 \mathrm{mg} /$ $\mathrm{dl}, \mathrm{C}$-reactive protein (CRP) of more than $2 \mathrm{mg} / \mathrm{dl}$, bilirubin of more than $2 \mathrm{mg} / \mathrm{dl}$, body mass index (BMI) of less than $18 \mathrm{~kg} / \mathrm{m}^{2}$, pregnancy, history of stroke, and patients receiving chemotherapy, anti-leukocyte drugs, TNF- $\alpha$ blockers, immunosuppressive drugs (e.g., tocilizumab) or with any diagnosed disease state that has produced leukopenia (e.g., acquired immune deficiency syndrome). Patient selection is shown in Fig. 1.

\section{Randomization}

Eligible patients were enrolled the day before surgery by one of the physicians involved in the study and randomly assigned into one of two groups (HA or control). Randomization was performed as block randomization by the online Randomizer for Clinical Trials 1.7.0 (https://www.meduniwien.ac.at/randomizer). To create homogenous and comparable groups, the randomization was stratified by sex and procedures.

\section{Outcomes}

\section{Primary outcome}

Primary outcomes were differences in the evolution of cytokines using the CytoSorb ${ }^{\mathrm{Tm}}$ adsorber for HA during cardiopulmonary bypass.

\section{Secondary outcomes}

Secondary outcomes were differences in LPS-induced release of TNF- $\alpha$; differences in the expression of HMGB1; changes in serum CRP or procalcitonin (PCT) concentrations; differences in the need of fluid components (crystalloid and colloid solutions), blood products (erythrocytes, fresh frozen plasma, and platelets), or catecholamine treatment; and changes in BIA, length of ICU stay, and 30-day mortality.

\section{Number of patients}

The fact that this is the first randomized controlled study and no prior data to cytokine level alterations in cardiac surgery patients using this HA device were available made us consider a mean difference of one standard 


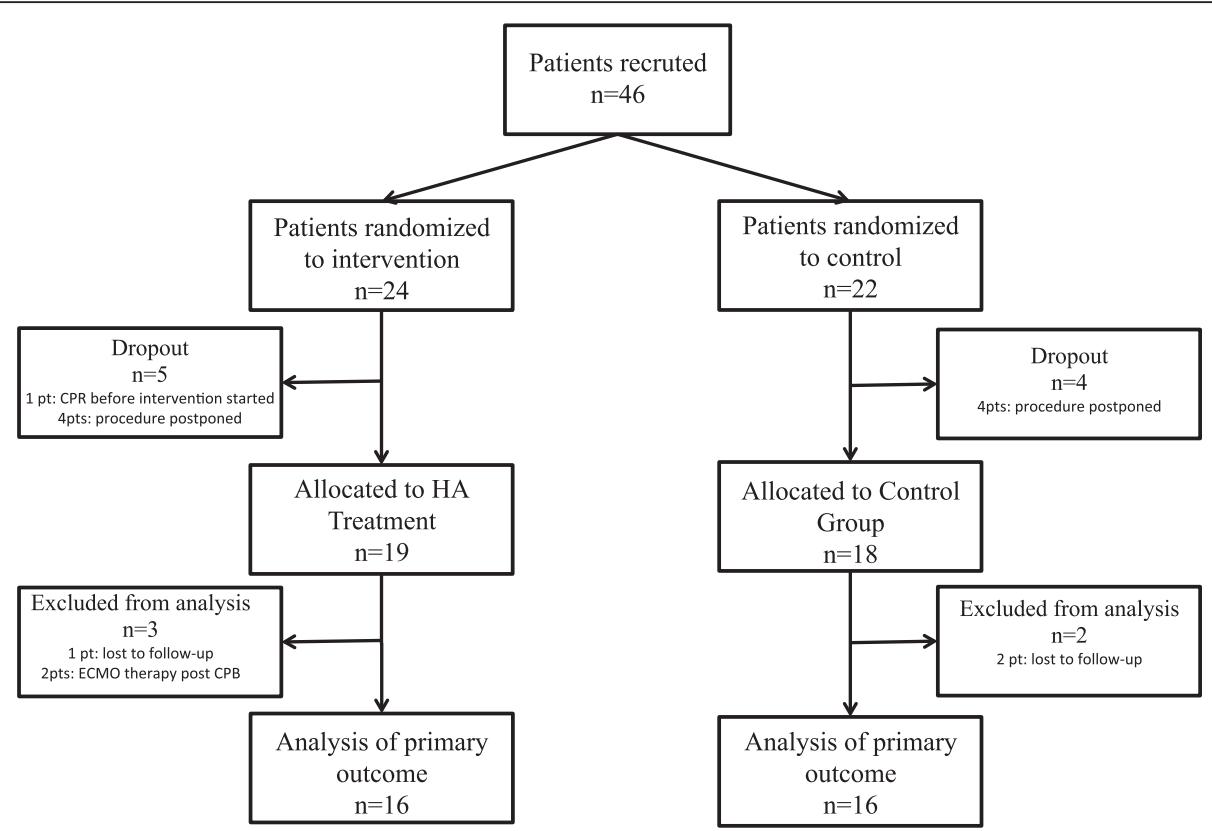

Fig. 1 The selection process for patients included in the study

deviation between groups as a clinically relevant effect. Under this assumption, we calculated by using a $t$ test a total of 16 individuals per group that are required to achieve $80 \%$ power with a significance level of $5 \%$. Therefore, we planned a total of 40 patients to allow an adequately powered analysis with $20 \%$ dropouts for complicated intraoperative course. To avoid the risk of low power, we increased the number of patients to 46 after the completion of the $36^{\text {th }}$ patient because a total of 7 patients dropped out at this time.

\section{Data collection}

Preoperative patient data (age, weight, height, sex, BIA, European system for cardiac operative risk evaluation [EuroSCORE], diagnosis, preoperative myocardial infarction within 24 hours $[\mathrm{MCI}]$, history of asthma bronchiale, chronic obstructive pulmonary disease [COPD], insulin or non-insulin-dependent diabetes mellitus [IDDM, NIDDM], history of chronic kidney disease [CKD], dialysis, left ventricular ejection fraction [LVEF], stable and unstable angina pectoris, cardial decompensation, peripheral arterial obstructive disease [PAOD], and arterial hypertension), surgery-related factors (kind of operation, duration of anaesthesia and surgery duration of $\mathrm{CPB}$ and aortic cross-clamp [AoCC], unplanned insertion of assist devices, amount of fluids [cristalloids or colloids], need of catecholamines [noradrenalin, dobutamin, levosimendan, vasopressin, or milrinon], and need of blood products [erythrocytes, fresh frozen plasma, or thrombocytes] or coagulation factors [fibrinogen, prothrombin complex concentrate, desmopressin, or recombinant factor VIIa]), intraoperative diuresis and postoperative data (use of catecholamines, BIA, length of stay on intensive care unit [LOS-ICU], length of mechanical ventilation, or need of extracorporeal membrane oxygenation [ECMO]) were collected by a case report form.

\section{Procedure}

Anaesthesia was induced and CPB circuit was primed (1000 ml crystalloid and $500 \mathrm{ml}$ colloid solution together with 5000 IE heparin, and $100 \mathrm{ml}$ mannitol $20 \%$ ) in accordance with institutional standards. $\mathrm{CPB}$ was performed by using non-pulsatile flow at $2.5 \mathrm{l} \cdot \mathrm{min}^{-1} \cdot \mathrm{m}^{-2}$, a non-heparin-coated circuit, and a membrane oxygenator (Quadrox ${ }^{\mathrm{Tm}}$, Maquet, Hirrlingen, Germany, or Capiox, Terumo, Eschborn, Germany). All study cases were performed by experienced cardiac anaesthesia fellows supervised by senior cardiac anaesthesiologists, both trained in transesophageal echocardiography (TOE), which was used to monitor myocardial performance and the impact of fluid loading and inotropic support on left and right ventricular function. Blood transfusion was performed in accordance with Society of Thoracic Surgeons/ Society of Cardiovascular Anesthesiologists (STS-SCA) transfusion guidelines [13, 14], and administration of coagulation factors was based predominantly on rotational thromboelastometry (ROTEM) variables and the coagulation profile of each patient.

In the intervention group, we installed the $300 \mathrm{ml}$ CytoSorb $^{\text {tu }}$ adsorber on the CPB machine. The active component of the CytoSorb ${ }^{\text {tw }}$ device consists of adsorbent polymer beads composed of porous polymerized 
divinylbenzene. These beads have pores that can adsorb hydrophobic molecules in a size range of approximately 10 to $55 \mathrm{kD}$, which is sufficient to remove almost all known cytokines. The polymer beads are encased in a polycarbonate canister commonly used in commercially available dialyzers. Blood was pumped actively through

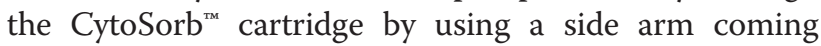
from the venous outflow tube and given back to the venous reservoir prior to the oxygenator. The flow through the cartridge was controlled by a roller pump with $200 \mathrm{ml} / \mathrm{min}$ to standardize flow conditions in all treated patients. The addition of $20 \mathrm{ml}$ crystalloid solution was necessary to fill the additional line in the treatment group. The control group was treated similarly, but no adsorber was installed.

\section{Blood sampling}

Blood samples were drawn in pyrogen-free vials, and plasma was separated by centrifugation and frozen $\left(-80{ }^{\circ} \mathrm{C}\right)$. Blood samples for cytokines (IL-1 $\beta$, IL-6, IL18 , TNF- $\alpha$, and IL-10) were determined at the following time points: A, before induction of anesthesia; $\mathrm{B}$, before $\mathrm{CPB}$; $\mathrm{C}$, at the end of $\mathrm{CPB}$; $\mathrm{D}, 2$ hours after $\mathrm{CPB}$; $\mathrm{E}$, 24 hours after CPB; F, 48 hours after CPB; and G, 120 hours after CPB. The ex vivo LPS-induced TNF- $\alpha$ production was measured at the time points $\mathrm{A}-\mathrm{C}, \mathrm{F}$, and G; and HMGB1 at time points B, D, and E. For the quantification of IL-1 $\beta$, IL-6, TNF- $\alpha$, and IL-10, we used the $\mathrm{BD}^{\mathrm{mm}}$ Cytometric Bead Array (CBA) Human Inflammatory Cytokines (BD Biosciences Europe, Erembodegem, Belgium) Kit; for quantification of IL-18, Human IL-18 Instant, ELISA (eBioscience, Inc., San Diego, CA, USA), and for quantification of HMGB1 the high-mobility group box 1 (HMGB1), ELISA Kit (MyBioSource, Inc., San Diego, CA, USA). For the measurement of ex vivo LPS-induced TNF- $\alpha$, lipopolysaccharide from Escherichia coli was purchased (Sigma-Aldrich GmbH, Vienna, Austria) and prepared. For the analysis of LPS-induced TNF- $\alpha$ release, Human TNF- $\alpha$ Instant, ELISA (eBioscience, Inc.) was performed on each sample. All analysis were conducted in accordance with the protocol of the manufacturer.

Blood samples for CRP, procalcitonin, albumine, fibrinogen, hemoglobin, thrombocytes, and leukocytes were determined at the following time points: a baseline value within 24 hours preoperatively (BL), 1st postoperative morning (1.POD), 2nd postoperative morning (2.POD), and 5th postoperative morning (5.POD).

\section{Bioelectrical impedance analysis}

We performed BIA by using $800 \mu \mathrm{A}$ at $50 \mathrm{kHz}$ with a single-frequency bioimpedance analyzer (Model BIA 101; Akern-RJL, Pontassieve, Italy). The skin was cleaned, and adhesive pregelled electrodes (Bianostic AT; Data-Input $\mathrm{GmbH}$, Wedemark, Germany) were placed on the hand (source on the third metacarpophalangeal joint and the detector on wrist, between the distal prominences of the radius and ulna) and the foot (source on the third metatarsophalangeal joint and the detector on the ankle, between the medial and lateral malleoli) of the right side while patients where in a recumbent position with the limbs abducted from the body. Measurements were performed within 24 hours preoperatively, 1.POD, 2.POD, and 5.POD. The measured BIA variables were resistance $(\mathrm{R})$, reactance $(\mathrm{Xc})$, the phase angle $(\arctan \mathrm{Xc} / \mathrm{R})$, and total body water (TBW). TBW was calculated by the following formulas according to the BIA analyzer we used $[15,16]$ :

Female : TBW $=0.382 *\left(\mathrm{Ht}^{2} / \mathrm{R}\right)+0.105 *$ weight +8.315 Male : TBW $=0.396 *\left(\mathrm{Ht}^{2} / \mathrm{R}\right)+0.143 *$ weight +8.399 .

\section{Statistical analysis}

Demographic and clinical baseline data were summarized by mean and standard deviation or mean and range, expressed through minimum and maximum, for metric variables or absolute frequencies for categorical variables. Differences between groups were analyzed by using the Student's $t$ test for continuous variables and Fisher's exact test for categorical variables.

The distributions of the cytokine levels were highly skewed; therefore, between-group differences of these variables were assessed by using the non-parametric Wilcoxon rank-sum test. The distributions were described by median and interquartile range (IQR) expressed through the first quartile and the third quartile.

The distributions of laboratory values were largely symmetric without severe outliers. These variables were described by mean and standard deviation, and the effect of HA was assessed by using analysis of covariance (ANCOVA) models. In these models, the outcome is explained by the treatment group (HA versus control), the stratification variables (procedure and sex), and the observed preoperative baseline value (except when analyzing the baseline differences). To describe the correlation between cytokine levels and duration of procedure, we calculated Spearman's rank correlation coefficients at the end of treatment time (time point $\mathrm{C}$ ).

The analysis of IL-10 suggests that the decrease after HA may follow an exponential function. To investigate this, we fitted the following model for time-dependent decay of IL-10 for each group by using the non-linear least squares method: mean IL-10 $=\mathrm{A}^{*} \exp \left(\lambda^{*}\right.$ time $)$. Also, to obtain a robust global test, we used a rerandomization test with 10,000 repeats. The patients were repeatedly randomly assigned anew with the same block randomization procedure as originally applied. In each repeat, a chi-squared-type statistic and a $P$ value were calculated as the proportion of 
resampled statistics being equal to or larger than the observed statistic.

\section{Results}

In total, 46 patients were included in the study and randomly assigned into one of two groups (HA or control). Nine patients (5 HA and 4 control) dropped out of the study after randomization: In six patients, the procedure was scheduled to another day or later in the day, when the study team was no longer available. One patient (HA) evolved a hemodynamical instability after skin incision leading to cardiopulmonary resuscitation and an acute onset of the $\mathrm{CPB}$, so no adsorber could be installed, and in two patients (1 HA and 1 control) we lost the follow-up of our main outcome measurements, so we excluded them too. Finally, we analyzed 37 patients; 19 patients were randomly assigned to the HA group and 18 patients to the control group. For the analysis of our primary outcome, we excluded the patients with unexpected post-treatment ECMO therapy $(n=2)$ because of differences in cytokine exaggeration (Fig. 1). Our patients had a mean age of $66 \pm 12$ years, the mean EuroSCORE was $5.4,30 \%$ of them were female, and the mean temperature during $\mathrm{CPB}$ was $33 \pm 2{ }^{\circ} \mathrm{C}$. All patients survived the 30-day period, except one patient (HA) who died on the $22^{\text {nd }}$ postoperative day because of multiple surgical complications. All other pre-, intra-, and post-operative patient characteristics showed no difference between both groups. Detailed results are shown in Table 1.

\section{Primary outcome}

We measured high amounts of IL-6 in both groups increasing after $\mathrm{CPB}$ and with a peak value 2 hours after CPB (HA: median 120.8, IQR 49.0-160.8 versus control: median 118.7, IQR 68.4-255.9, pg/ml, $P=0.6781$ ). One patient (control) showed an increase of IL-6 after skin incision $(2.1 \mathrm{pg} / \mathrm{ml})$; in all other patients, the activation started during $\mathrm{CPB}$. No significant difference was found between the treatment and control group for all time points. The correlations for IL- 6 and the end of treatment duration were 0.34 for the HA group and 0.46 for the control group.

For IL-10, we observed an increase in one patient (HA) after inducing anesthesia $(11.3 \mathrm{pg} / \mathrm{ml})$. In four patients (2 HA and 2 control), the activation of IL-10 started before CPB; one of those also had an increase of IL-6. We did not find any similarities in those patients with pre-CPB increased levels of cytokines. IL-10 reached a peak value at the end of CPB (HA: median 13.1, IQR 3.3-18.7 versus control: median 18.5, IQR 5.7-68.0 pg/ml, $P=0.1562)$. The decrease of IL-10 seems to be earlier in the control group showing significant differences 24 hours after CPB (HA: median 0.3,
IQR $0-4.5 \mathrm{pg} / \mathrm{ml}$ versus control: median $0, P=0.0347$ ) and 48 hours after CPB (HA: median 0, IQR $0-1.2 \mathrm{pg} /$ $\mathrm{ml}$ versus control: not traceable, $P=0.0185)$. The correlations for IL-10 and the end of treatment time were 0.02 for the HA group and 0.32 for the control group.

The exponential decay model matched rather well the observed mean values and showed different characteristics for the two groups $(P=0.0188)$. For detailed results of the analysis of the cytokine evolution, see Table 2 and Figs. 2 and 3.

We detected traceable values in only two patients for TNF- $\alpha$ and IL-18 and in one patient for IL- $1 \beta$ over the perioperative period. Therefore, we did not perform any statistical analysis on TNF- $\alpha$, IL-18, and IL-1 $\beta$.

Owing to technical problems with thawing of our frozen blood samples, we additionally lost one patient in the HA group and two patients in the control group. So we were able to analyze our primary outcome in only 16 patients in the HA group and 16 patients in the control group (Fig. 1).

\section{Secondary outcome parameters}

Ex vivo LPS-induced TNF- $\alpha$ exaggeration could be stimulated at all determined time points. We observed a significant difference between both groups preoperatively (HA: median 2216, IQR 1742-2659 versus control: median 3364, IQR $2579-4893 \mathrm{pg} / \mathrm{ml}, P=0.004$ ) and a reduction of LPS-induced TNF- $\alpha$ after CPB but no significant difference between both groups. On the 2.POD, LPSinduced TNF- $\alpha$ reached again preoperative values but showed a significant lower amount in the HA group (HA: median 788, IQR 679-1272 versus control: median 3959, IQR $2088-4777 \mathrm{pg} / \mathrm{ml}, P=0.0115$ ) as well as on 5.POD (HA: median 1737, IQR 843-2535 versus control: median 3358, IQR 3017-3672 pg/ml, $P=0.0205)$. Unfortunately, we were able to analyze only 18 (9 HA and 9 control) patients on 2.POD and 17 (9 HA and 8 control) patients on 5.POD (Table 2).

HMGB1 showed a significantly different expression at baseline before treatment (HA: median 0, IQR 0-28.1 versus control: median 48.6 , IQR $12.7-597.3 \mathrm{pg} / \mathrm{ml}$, $P=0.0208$ ) but no differences in the period after $\mathrm{CPB}$, although post-treatment maximum levels in the control group were nearly double that of the HA group (HA: 705 versus control: $1594 \mathrm{pg} / \mathrm{ml}$ ). The post hoc analysis was possible in 29 patients (15 HA and 14 control). We did not observe any differences in other inflammation markers like CRP or PCT or differences in leukocytes, thrombocytes, hemoglobin, albumin, or fibrinogen levels. The analysis of differences before and after intervention within the groups resulted in significant decreases in hemoglobin, albumin, and thrombocytes, according to hemodilution, as well as significant increases in CRP and leukocytes, according to usual 
Table 1 Patient and surgical characteristics

\begin{tabular}{|c|c|c|c|c|}
\hline & $\mathrm{HA}(\mathrm{n}=19)$ & Control $(n=18)$ & OR (95 \% Cl) & $P$ value \\
\hline \multicolumn{5}{|l|}{ Preoperative characteristics } \\
\hline Age, years & $64(30-81)$ & $69(51-81)$ & & 0.1737 \\
\hline Male & 12 & 14 & $0.50(0.09,2.56)$ & 0.4756 \\
\hline Female & 7 & 4 & & \\
\hline $\mathrm{BMI}, \mathrm{kg} / \mathrm{m}^{2}$ & $27(18-35)$ & $27(20-39)$ & & 0.6593 \\
\hline EuroSCORE & $4.0 \pm 3.6$ & $6.0 \pm 4.6$ & & 0.154 \\
\hline Resistance & $411.7 \pm 210.2$ & $442.7 \pm 101.6$ & & 0.6948 \\
\hline Phase angle & $5.1 \pm 1.5$ & $4.9 \pm 0.7$ & & 0.7078 \\
\hline TBW & $56.3 \pm 14.1$ & $46.6 \pm 11.3$ & & 0.1205 \\
\hline $\mathrm{MCl}$ & 0 & 0 & & 1 \\
\hline Asthma & 0 & 0 & & 1 \\
\hline COPD & 3 & 3 & $0.94(0.11-8.15)$ & 1 \\
\hline NIDDM & 6 & 4 & $1.60(0.30-9.56)$ & 0.714 \\
\hline IDDM & 0 & 2 & $0(0,4.99)$ & 0.2297 \\
\hline CKD & 0 & 0 & & 1 \\
\hline Cardiac decompensation & 2 & 1 & $1.96(0.09-124.72)$ & 1 \\
\hline PAOD & 3 & 1 & $3.10(0.22-176.81)$ & 0.6039 \\
\hline Art. hypertension & 10 & 10 & $0.89(0.20-3.90)$ & 1 \\
\hline Dialysis & 0 & 0 & & 1 \\
\hline Angina pectoris (absence of) & 16 & 13 & & 0.5392 \\
\hline Angina pectoris (stable) & 3 & 4 & & \\
\hline Angina pectoris (instable) & 0 & 1 & & \\
\hline LVEF $>50 \%$ & 12 & 13 & & 0.4756 \\
\hline LVEF $30-50 \%$ & 7 & 4 & & \\
\hline LVEF $<30 \%$ & 0 & 1 & & \\
\hline \multicolumn{5}{|l|}{ Intraoperative characteristics } \\
\hline Valve procedure, M/F & $7 / 4$ & $7 / 4$ & & 0.4819 \\
\hline$C A B G, M / F$ & $2 / 1$ & $5 / 0$ & & \\
\hline Combined procedure, M/F & $3 / 2$ & $2 / 0$ & & \\
\hline Anesthesia time, min & $474(290-673)$ & $427(277-570)$ & & 0.1683 \\
\hline Surgery time, min & $369(219-630)$ & $327(225-493)$ & & 0.2152 \\
\hline CPB time, $\min$ & $191(112-288)$ & $170(83-274)$ & & 0.2064 \\
\hline AoCC time, min & $138(57-242)$ & $117(36-179)$ & & 0.1423 \\
\hline Fibrinogen, $\mathrm{g}$ & $1.7(0-5)$ & $0.8(0-4)$ & & 0.077 \\
\hline Thrombocytes, units & $0.15(0-1)$ & $0.2(0-1)$ & & 0.6304 \\
\hline PCC, IE & $605(0-2000)$ & $389(0-3000)$ & & 0.415 \\
\hline FFP, units & $0.5(0-9)$ & 0 & & 0.3306 \\
\hline Erythrocytes, units & $1.4(0-7)$ & $0.7(0-3)$ & & 0.2021 \\
\hline Cristalloids, ml & $5239 \pm 1569$ & $4311 \pm 1230$ & & 0.0526 \\
\hline Colloids, ml & $571 \pm 327$ & $514 \pm 130$ & & 0.4873 \\
\hline Diuresis & $826 \pm 352$ & $978 \pm 589$ & & 0.3516 \\
\hline \multicolumn{5}{|l|}{ Postoperative characteristics } \\
\hline LOS-ICU, days & $2.3 \pm 2.0$ & $2.4 \pm 1.9$ & & 0.8721 \\
\hline Mechanical ventilation, days & $0.7 \pm 1.6$ & $0.2 \pm 0.4$ & & 0.19 \\
\hline
\end{tabular}


Table 1 Patient and surgical characteristics (Continued)

\begin{tabular}{|c|c|c|c|}
\hline Balance 1POD & $9374 \pm 4785$ & $7303 \pm 2664$ & 0.1124 \\
\hline vaECMO, n & 2 & 0 & 0.4865 \\
\hline 30-day mortality & 1 & 0 & 1 \\
\hline
\end{tabular}

postoperative inflammation, within both groups. We did not find differences in the change of PCT, HMGB1, or fibrinogen levels within both groups. Detailed information is shown in Tables 3 and 4.

We performed a BIA in 19 patients $(9 \mathrm{HA}$ and 10 control). However no differences in baseline values in resistance, phase angle, or TBW were observed between both groups (Table 5).

We also analyzed the need of catecholamines in both groups within the first 24 hours, but we did not analyze differences on day 2 or 5 , because only 20 patients (9 HA and 11 control) remained in the ICU after 24 hours. We did not find any differences in the need of noradrenalin, dobutamin, or levosimendan, although it is worth mentioning that only a total of eight patients received dobutamin in the control group and 11 patients in the HA group, as well as only eight (5 HA and 3 control) patients were treated with levosimendan (Table 6).

\section{Discussion}

Cardiac surgery is associated with an unpredictable activation of the immune system with an increase of proinflammatory cytokines as well as a decrease of antiinflammatory cytokines, which is caused by blood contact with artificial surfaces and therefore is linked to adverse outcomes $[17,18]$. In this (to our knowledge) first controlled study in patients undergoing on-pump cardiac surgery treated with the CytoSorb ${ }^{\mathrm{Tm}}$ adsorber, no significant differences of pro-inflammatory cytokine levels were found. Even though a reduction of absolute levels within the first 24 hours after CPB is noticeable, no significant changes were observed. Given that we did not observe any adverse device-related side effects or differences in reduction of blood cells or albumin, our study shows that using the CytoSorb ${ }^{\text {tut }}$ adsorber cartridge in a CPB circuit is technically feasible.

The indication for the CytoSorb ${ }^{\text {Tn }}$ adsorber is to reduce cytokine concentrations in various clinical situations with elevated cytokine levels and has been tested previously and demonstrated significant cytokine adsorption [19-21], an effect we could not reproduce in our patients. There may be numerous reasons for that outcome: First, we had a mean treatment time of $191 \pm 56$ minutes, which may be too short to allow a significant reduction of cytokine levels, although we did not find any correlation between cytokine peaks and treatment time. In all previously conducted studies [19-21] and case reports [12, 22, 23], the treatment time was at least 4 hours up to 4 days. In a CPB-porcine model with 5 hours of treatment time, also no effect in IL-6 or TNF- $\alpha$ has been found [24]. Secondly, we effectively observed a CPB-triggered immunoactivation and an increase in cytokines and inflammation markers after CPB and therefore after HA treatment. So it might be owing to a concentration-dependent adsorption of CytoSorb $^{\text {tw }}$ that we did not observe any HA of cytokines. Third, we may have expected a too optimistic effect and therefore we may have planned a too small sample size, a fact which is also shown by the observed strong inter-individual differences in the amounts of cytokine levels. But at the time of planning this pilot study, no clinically relevant data concerning our primary outcome were available. And, fourth, we included only the least sick cohort of patients undergoing cardiac surgery. Although we did not restrict the EuroSCORE levels of our included patients, our exclusion criteria resulted in a moderate preoperative risk for postoperative outcomes. In a recently published cohort analysis [25] representing more than 9,000 cardiac surgical patients operated at our center, we found similar demographics, so that the population we investigated represents in our opinion a cross-section of elective moderate-risk patients operated at our center.

We rarely observed the production of TNF- $\alpha$ in our patients and this goes hand in hand with the contentious role of TNF- $\alpha$ within $\mathrm{CPB}$; although some studies have shown an increase, others have not [26]. Also, IL-1 $\beta$ has been found to be detectable in only a small proportion of patients with systemic inflammatory response syndrome and sepsis [27].

IL-10 is thought to downregulate cytokine production [27], and high concentrations have been observed in our patients. Our results follow a similar time course to the pro-inflammatory cytokines with an early peak and subsequently falling concentrations. Interestingly, we observed a slower decrease of postoperative IL-10 levels in the HA group and therefore a longer effect up to 48 hours postoperatively. Recently, higher IL-10 levels following cardiac surgery have been associated with a decreased risk of mortality [28]. Although we 
Table 2 Comparison of cytokine levels

\begin{tabular}{|c|c|c|c|c|c|c|c|c|}
\hline & Treatment & Preoperative & Before CPB & After CPB & 2 hours after $C P B$ & 24 hours after $C P B$ & 48 hours after $C P B$ & 5.POD \\
\hline \multirow[t]{3}{*}{$\mathrm{IL}-6, \mathrm{pg} / \mathrm{ml}$} & $\mathrm{HA}$ & Not traceable & Not traceable & $62.9(10.8,98.7)$ & $120.8(49.0,160.8)$ & $111.6(53.7,253.5)$ & $89.0(61.4,160.5)$ & $0.4(0,8.3)$ \\
\hline & Control & Not traceable & 0 & $63.6(41.2,154.9)$ & $118.7(68.4,255.9)$ & $120.9(68.0,198.5)$ & $67.7(43.7,134.5)$ & $8.2(0.8,19.4)$ \\
\hline & $P$ value & & 0.3485 & 0.3267 & 0.6781 & 0.9837 & 0.3809 & 0.0999 \\
\hline \multirow[t]{3}{*}{ |L-10, pg/ml } & $\mathrm{HA}$ & 0 & 0 & $13.1(3.3,18.7)$ & $5.9(0.5,12.9)$ & $0.3(0,4.5)$ & $0(0,1.2)$ & 0 \\
\hline & Control & Not traceable & 0 & $18.5(5.7,68.0)$ & $2.3(0,11.6)$ & 0 & Not traceable & Not traceable \\
\hline & $P$ value & 0.3485 & 1.0 & 0.1562 & 0.6743 & 0.0347 & 0.0185 & 0.3485 \\
\hline \multirow[t]{3}{*}{ TNFa-LPS, pg/ml } & $\mathrm{HA}$ & $2216(1742,2659)$ & $2534(914,3023)$ & $40(1,82)$ & Non fec. & Non fec. & $788(679,1272)$ & $1737(843,2535)$ \\
\hline & Control & $3364(2579,4893)$ & $3403(1679,4394)$ & $77(34,316)$ & Non fec. & Non fec. & $3959(2088,4777)$ & $3358(3017,3672)$ \\
\hline & $P$ value & 0.004 & 0.1378 & 0.2049 & & & 0.0115 & 0.0205 \\
\hline \multirow[t]{3}{*}{ HMGB1, pg/ml } & $\mathrm{HA}$ & Non fec. & $0(0,28.1)$ & Non fec. & $5.7(0,34.2)$ & $0(0,72.5)$ & Non fec. & Non fec. \\
\hline & Control & Non fec. & $48.6(12.7,597.3)$ & Non fec. & $41.5(4.5,266.9)$ & $26.2(0,281,2)$ & Non fec. & Non fec. \\
\hline & $P$ value & & 0.0208 & & 0.1327 & 0.3724 & & \\
\hline
\end{tabular}

Values are presented as median (first quartile, third quartile). The listed $P$ values were calculated by using Wilcoxon rank-sum tests. Abbreviations: CPB, cardiopulmonary bypass; HA, hemoadsorption; HMBG1,

high-mobility group box 1; IL, interleukin; fec., fecit; POD, postoperative day; TNF-a, tumor necrosis factor-alpha; TNFa-LPS, lipopolysaccharide-induced TNF-a 


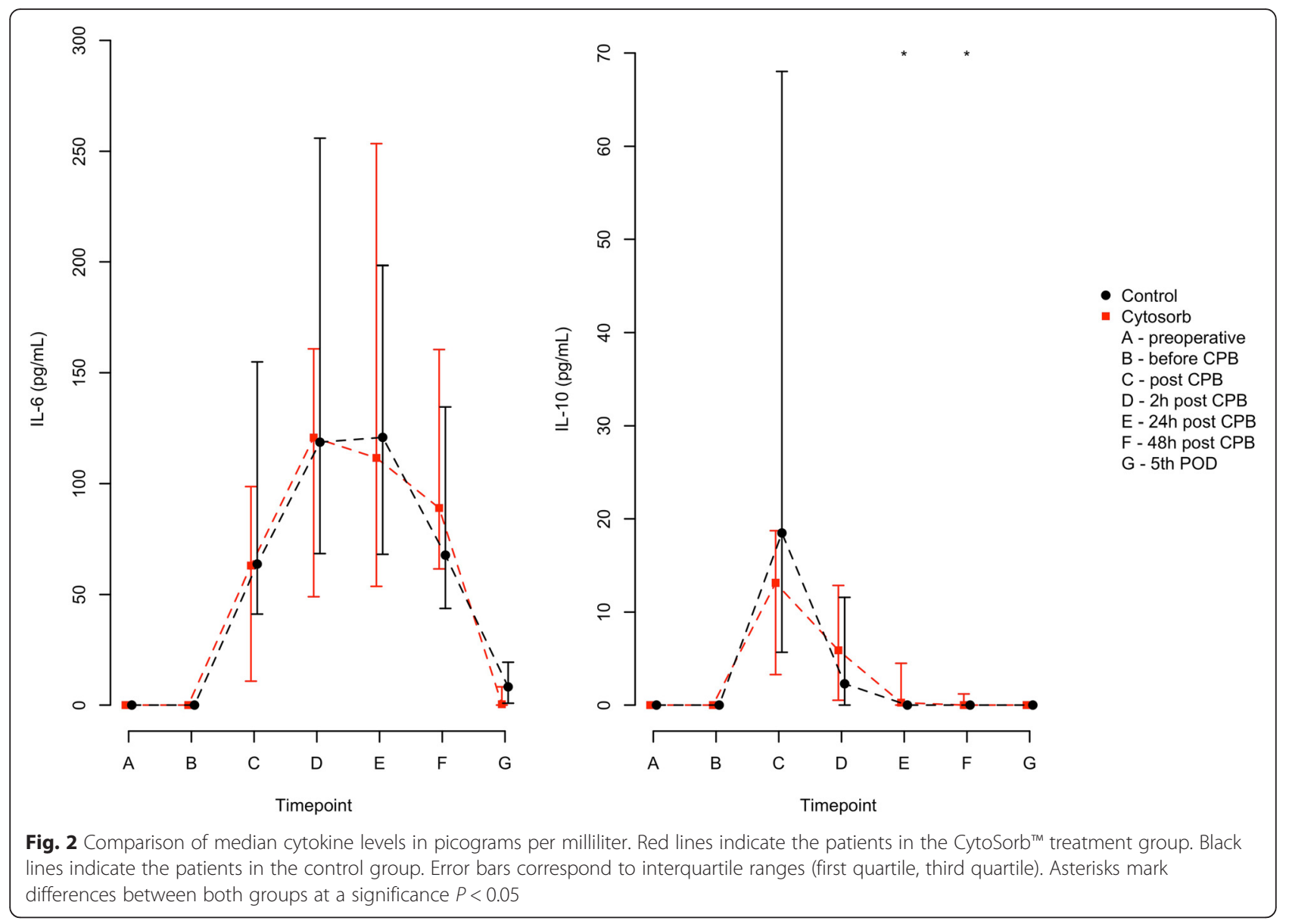

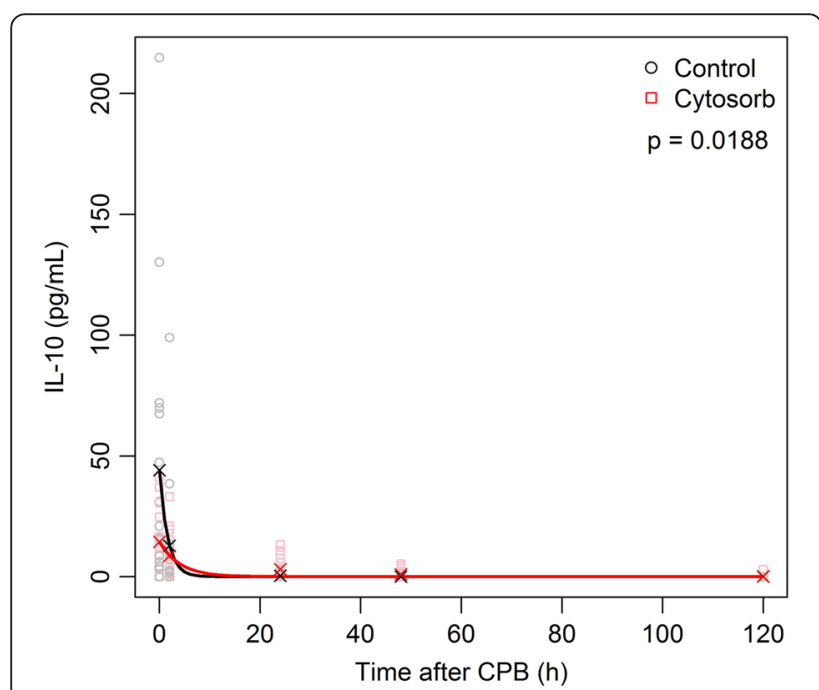

Fig. 3 Exponential decay model for mean interleukin-10 (IL-10). Black circles indicate IL-10 values for the control group, and red squares indicate IL-10 values for the CytoSorb ${ }^{\text {TM }}$ group did not find a reduction in mortality, this arguable immuno-protective effect needs to be investigated further.

We also found significant time-dependent changes in ex vivo LPS-induced TNF- $\alpha$ release. Surprisingly, we had observed a lower stimulation rate in the HA group already before treatment. That could be based on an effect modification of comorbidities like diabetes mellitus (DM). There is good evidence that patients with DM are associated with an immunosuppressive condition and have poorer humoral response, including decreased cytokine production, and therefore have an increased susceptibility to infections [29-31]. Although there were no differences between both groups, the lowest levels of LPS-induced TNF- $\alpha$ release were found in HA-treated patients with DM. Additionally, it may be an effect of an associated, preoperative drug therapy such as metformin, aspirin, or statins, which we did not record. HMGB1 is associated with the inflammatory response after ischemia/ reperfusion injury after cardiopulmonary bypass, and it has been shown that a reduction decreases the markers indicating cardiac damage. Also, elevated levels of HMGB1 have been correlated with the disease severity of heart failure [32-35]. However, we observed preoperative 
Table 3 Comparison of laboratory values

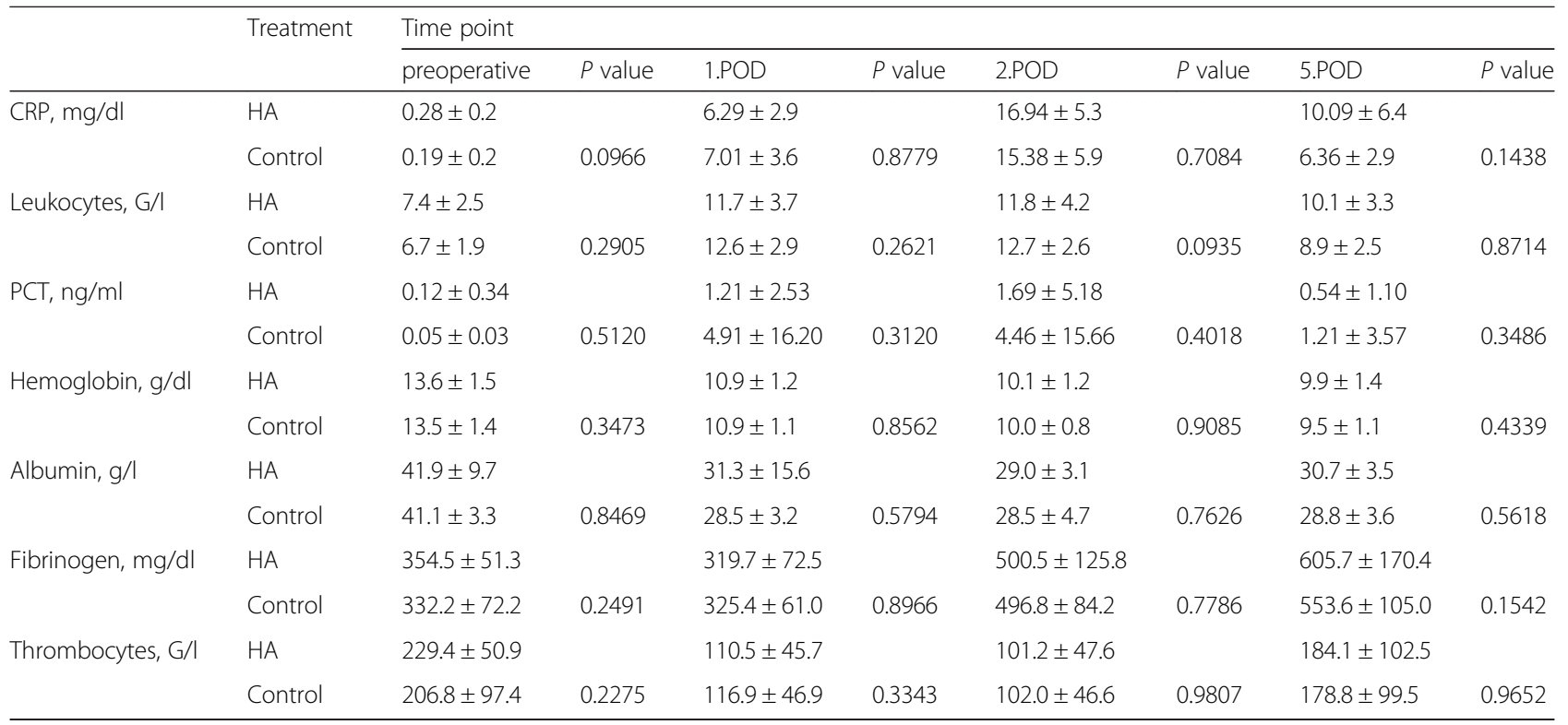

Values are presented as mean \pm standard deviation. The listed $P$ values of statistical tests were calculated by using analysis of covariance. Abbreviations: CRP, $\mathrm{C}-$ reactive protein; $\mathrm{HA}$, hemoadsorption; $\mathrm{PCT}$, procalcitonin; $\mathrm{POD}$, postoperative day

differences in HMGB1; therefore, it is not possible to interpret the results if there is a post-treatment difference. According to our LPS-induced TNF- $\alpha$ results, we cannot rule out that there is a hidden phenotypic difference between groups despite randomization that may be

Table 4 Differences between preoperative and postoperative values

\begin{tabular}{llcll}
\hline & Treatment & Mean difference & $95 \% \mathrm{Cl}$ & $P$ value \\
\hline CRP, mg/dl & HA & 6.0 & $-7.4,-4.6$ & $<0.0001$ \\
& Control & 6.8 & $-8.6,5.0$ & 0.0002 \\
Leukocytes, G/l & HA & 4.24 & $-5.82,-2.66$ & 0.0115 \\
& Control & 5.86 & $-7.47,-4.25$ & $<0.0001$ \\
PCT, ng/ml & HA & 1.08 & $-2.24,0.07$ & 0.0649 \\
& Control & 5.07 & $-13.64,3.49$ & 0.2271 \\
Hemoglobin, g/dl & HA & -2.7 & $2.0,3.3$ & $<0.0001$ \\
& Control & -2.7 & $2.0,3.4$ & $<0.0001$ \\
Albumin, g/l & HA & -10.5 & $2.1,19.0$ & 0.0175 \\
& Control & -12.6 & $11.2,14.0$ & $<0.0001$ \\
Fibrinogen, mg/dl & HA & -34.8 & $-1.7,71.3$ & 0.0607 \\
& Control & -6.8 & $-22.6,36.2$ & 0.6332 \\
Thrombocytes, G/l & HA & -119.0 & $99.2,138.7$ & 0.0006 \\
& Control & -89.8 & $60.0,119.7$ & $<0.0001$ \\
& HA & 9.5 & $-38.3,57.3$ & 0.6764 \\
HMGB 1, pg/ml & Control & 64.2 & $-70.9,199.2$ & 0.3235 \\
\hline
\end{tabular}

Values are presented as mean difference and $95 \%$ confidence interval (Cl). The listed $P$ values of statistical tests were calculated by using paired $t$ tests. Abbreviations: CRP, C-reactive protein; HA, hemoadsorption; HMGB1, high-mobility box group; PCT, procalcitonin associated with higher levels of HMGB1 before CPB. But we think the effects of HMGB1 and HA on patients' postoperative course should be investigated further.

Last, we observed neither any differences in our patients' body composition nor any need of catecholamines or postoperative fluid balances. Therefore, we cannot conclude that there is less edema formation in the HA group. Unfortunately, we did not record systemic vascular resistance after CPB to assess that the HA group was more vasodilated.

A limitation of a study such as ours may be effect modifications owing to omitted or unobserved confounding risk indicators, although we included the most relevant risk indicators to rule out any systematic effect. However, we did not monitor the use of non-steroidal anti-inflammatory drugs preoperatively (e.g., aspirin), statins, or metformin, which may have anti-inflammatory effects [36, 37]. Another limitation of our study can be that our study design was not double-blinded, only blinded in patients. Although blinding in studies involving operative management and medical devices is rarely feasible, we do not think that would have affected the outcome. Additionally, owing to technical problems, we were not able to perform BIA in every patient, and we have to report that our BIA monitor is validated in healthy subjects up to only 66 years [16]. Last, a limitation of the study is that we did not observe the cytokine levels before and after the HA cartridge and therefore we cannot be sure what proportion of blood has been treated. We can only estimate that despite the small amount of 3 to $4 \%$ total blood volume purified each 
Table 5 Comparison of bioelectrical impedance analysis

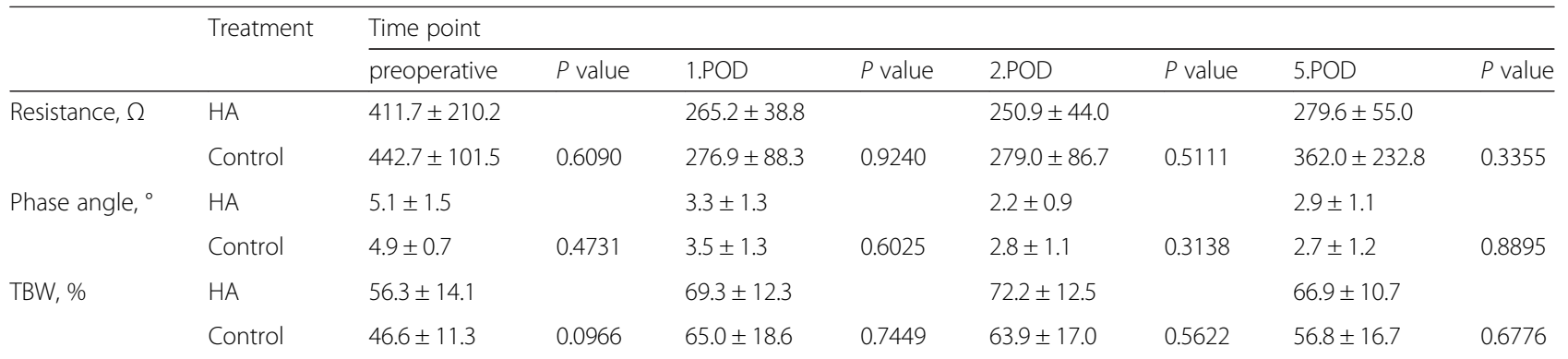

Values are presented as mean \pm standard deviation. The listed $P$ values of statistical tests were calculated by using analysis of covariance. Abbreviations: HA, hemoadsorption; POD, postoperative day; TBW, total body water

minute, more than $99 \%$ of the blood volume has passed through the HA device after our mean treatment time of 191 minutes.

\section{Conclusions}

We claim that the use of the CytoSorb ${ }^{\text {mo }}$ adsorber cartridge in a CPB circuit is technically feasible. We observed a longer-lasting anti-inflammatory effect of IL-10 in the HA group, which needs to be investigated further. We did not observe significantly relevant changes in the evolution of pro-inflammatory cytokines in patients treated with the CytoSorb ${ }^{\text {max }}$ adsorber device during CPB. However, we did not find any effects on our patients' clinical outcomes, although future studies should endeavour to increase the sample size. By reason of several involved pathways in the complex pathogenesis of the inflammatory reaction to $\mathrm{CPB}$, the inhibition of a single pathway may not achieve sufficient inhibition of the entire pro-inflammatory cascade to significantly improve clinical outcomes [38]. We found an inhomogeneous inflammatory response expressed by high inter-individual differences in cytokine levels between our patients. A greater homogeneity may be achieved by identifying those patients or procedures, which are triggering an exaggerated inflammatory response to $\mathrm{CPB}$ like patients with endocarditis, procedures on the aortic arch needing hypothermic cardiac arrest or transplant surgery. This hypothesis requires further investigations.

Table 6 Catecholamine treatment

\begin{tabular}{|c|c|c|c|c|c|c|c|c|c|c|}
\hline & \multicolumn{2}{|c|}{ Treatment } & \multicolumn{8}{|l|}{ Time point } \\
\hline & & & before CPB & $P$ value & After CPB & $P$ value & 2 hours after CPB & $P$ value & 1.POD & $P$ value \\
\hline \multirow[t]{6}{*}{ Noradrenalin, $\mu \mathrm{g} \mathrm{kg}^{-1} \mathrm{~min}^{-1}$} & Control & Mean \pm SD & $0.03 \pm 0.04$ & & $0.13 \pm 0.11$ & & $0.08 \pm 0.08$ & & $0.06 \pm 0.10$ & \\
\hline & & Max & 0.12 & & 0.36 & & 0.30 & & 0.36 & \\
\hline & & $N$ & 13 & & 18 & & 16 & & 8 & \\
\hline & $\mathrm{HA}$ & Mean \pm SD & $0.04 \pm 0.05$ & & $0.13 \pm 0.15$ & & $0.14 \pm 0.20$ & & $0.07 \pm 0.14$ & \\
\hline & & Max & 0.18 & & 0.62 & & 0.74 & & 0.6 & \\
\hline & & N & 14 & 0.3138 & 18 & 0.8503 & 14 & 0.3075 & 7 & 0.8771 \\
\hline \multirow[t]{6}{*}{ Dobutamin, $\mu \mathrm{g} \mathrm{kg}{ }^{-1} \mathrm{~min}^{-1}$} & Control & Mean \pm SD & $0.15 \pm 0.50$ & & $1.78 \pm 2.38$ & & $1.50 \pm 2.05$ & & $1.18 \pm 1.64$ & \\
\hline & & Max & 1.72 & & 6.94 & & 5.55 & & 5.00 & \\
\hline & & $N$ & 3 & & 8 & & 8 & & 8 & \\
\hline & $\mathrm{HA}$ & Mean \pm SD & $0.05 \pm 0.23$ & & $2.30 \pm 2.42$ & & $1.28 \pm 1.51$ & & $1.32 \pm 1.58$ & \\
\hline & & Max & 1.00 & & 8.00 & & 4.1 & & 5.34 & \\
\hline & & $\mathrm{N}$ & 1 & 0.4163 & 11 & 0.5136 & 10 & 0.7134 & 10 & 0.7871 \\
\hline \multirow[t]{6}{*}{ Levosimendan, $\mathrm{\mu g} \mathrm{kg}^{-1} \mathrm{~min}^{-1}$} & Control & Mean \pm SD & 0 & & $0.03 \pm 0.07$ & & $0.03 \pm 0.07$ & & $0.01 \pm 0.03$ & \\
\hline & & Max & 0 & & 0.2 & & 0.2 & & 0.1 & \\
\hline & & N & 0 & & 3 & & 3 & & 2 & \\
\hline & $\mathrm{HA}$ & Mean \pm SD & $0.02 \pm 0.05$ & & $0.07 \pm 0.18$ & & $0.04 \pm 0.08$ & & $0.01 \pm 0.03$ & \\
\hline & & Max & 0.20 & & 0.74 & & 0.2 & & 0.12 & \\
\hline & & N & 2 & 0.1868 & 5 & 0.3325 & 5 & 0.5228 & 1 & 0.6963 \\
\hline
\end{tabular}




\section{Key messages}

- Using the CytoSorb ${ }^{\mathrm{Tw}}$ adsorber during cardiopulmonary bypass did not change patients' perioperative course.

- Activation of cytokines due to cardiopulmonary bypass is inhomogeneous and shows high inter-individual differences between our patients. Patients with an exaggerated inflammatory response to cardiac surgery need to be identified.

- A longer-lasting anti-inflammatory effect of IL-10 was observed in patients who were treated with hemadsorption.

- The use and installation of the CytoSorb ${ }^{\text {тx }}$ adsorber on CPB were technically feasible, and no adverse device-related side effects occurred.

\begin{abstract}
Abbreviations
ACT: Activated clotting time; aHTN: Arterial hypertension; AoCC: Aortic cross-clamp; AP: Angina pectoris; BIA: Bioelectrical impedance analysis; BIS: Bispectral index; BMI: Body mass index; CABG: Coronary artery bypass graft; CKD: Chronic kidney disease; COPD: Chronic obstructive pulmonary disease; CPB: Cardiopulmonary bypass; CPR: Cardiopulmonary resuscitation; CRP: C-reactive protein; ECMO: Extracorporeal membrane oxygenation; EuroSCORE: European system for cardiac operative risk evaluation; HA: Hemoadsorption; HMGB1: High-mobility group box 1; IDDM: Insulin-dependent diabetes mellitus; IL: Interleukin; LOS-ICU: Length of stay in the intensive care unit; LPS: Lipopolysaccharide; LVEF: Left ventricular ejection fraction; MCI: Myocardial infarction; NIDDM: Non-insulin-dependent diabetes mellitus; PAOD: Peripheral arterial obstructive disease;

PCC: Prothrombin complex concentrate; PCT: Procalcitonin; POD: Postoperative
\end{abstract} day; TBW: Total body water; TNF-a: Tumor necrosis factor-alpha.

\section{Competing interests}

$\mathrm{MHB}$ and $\mathrm{MJH}$ have received travel funding for a lecture from CytoSorbents Europe $\mathrm{GmbH}$. All other authors declare that they have no competing interests.

\section{Authors' contributions}

MHB contributed to conception and design, to drafting of the article, to critical revision of the article for important intellectual content, to final approval of the article, and to laboratory work, patient recruitment, and follow-up investigations. FH contributed to conception and design. MJH and AS contributed to conception and design, to drafting of the article, to critical revision of the article for important intellectual content, and to final approval of the article. RR contributed to drafting of the article and to critical revision of the article for important intellectual content and provided statistical expertise. HR and DW contributed to critical revision of the article for important intellectual content and to laboratory work, patient recruitment, and follow-up investigations. $\mathrm{KD}, \mathrm{PO}, \mathrm{CL}$, and FP contributed to laboratory work, patient recruitment, and follow-up investigations. All authors read and approved the final manuscript.

\section{Acknowledgments}

We thank all the medical staff from the Department of Cardiothoracic and Vascular Anesthesia and the Department for Cardiac Surgery for contributing to the study. Special thanks go to our medical students David Hirschl and Christoph Steinkellner for their invaluable help in data collection.

\section{Disclosure of funding}

Materials for this study have been partly financially supported by CytoSorbents Europe $\mathrm{GmbH}$. All other sources of funding for laboratory measurements and human resources were departmental and institutional funding.

\section{Author details}

'Department of Cardiothoracic and Vascular Anaesthesia and Intensive Care Medicine, Medical University of Vienna, Waehringer Guertel 18-20, A-1090 Vienna, Austria. ${ }^{2}$ Department of Surgery, Research Laboratories, Medical University of Vienna, Lazarettgasse 14, A-1090 Vienna, Austria. ${ }^{3}$ Centre for Medical Statistics, Informatics and Intelligent Systems, Medical University of Vienna, Spitalgasse 23, A-1090 Vienna, Austria. ${ }^{4}$ Department of Cardiac Surgery, Medical University of Vienna, Waehringer Guertel 18-20, A-1090 Vienna, Austria. ${ }^{5}$ Core Facilities, Core Facility Flow Cytometry, Medical University of Vienna, Lazarettgasse 14, 1090 Vienna, Austria.

Received: 16 December 2015 Accepted: 22 March 2016

Published online: 09 April 2016

\section{References}

1. Tomic V, Russwurm S, Moller E, Claus RA, Blaess M, Brunkhorst F, et al. Transcriptomic and proteomic patterns of systemic inflammation in on-pump and off-pump coronary artery bypass grafting. Circulation. 2005;112:2912-20.

2. Diegeler A, Doll N, Rauch T, Haberer D, Walther T, Falk V, et al. Humoral immune response during coronary artery bypass grafting: A comparison of limited approach, "off-pump" technique, and conventional cardiopulmonary bypass. Circulation. 2000;102(19 Suppl 3):III95-III100.

3. Chew MS, Brandslund I, Brix-Christensen V, Ravn HB, Hjortdal VE, Pedersen J, et al. Tissue injury and the inflammatory response to pediatric cardiac surgery with cardiopulmonary bypass: a descriptive study. Anesthesiology. 2001;94:745-53. discussion 5A.

4. de Jong PR, Schadenberg AW, van den Broek T, Beekman JM, van Wijk F, Coffer PJ, et al. STAT3 regulates monocyte TNF-alpha production in systemic inflammation caused by cardiac surgery with cardiopulmonary bypass. PLoS One. 2012;7:e35070.

5. Shen X, Li WQ. High-mobility group box 1 protein and its role in severe acute pancreatitis. World J Gastroenterol. 2015;21:1424-35.

6. Seghaye M, Duchateau J, Bruniaux J, Demontoux S, Bosson C, Serraf A, et al. Interleukin-10 release related to cardiopulmonary bypass in infants undergoing cardiac operations. J Thorac Cardiovasc Surg. 1996;111:545-53.

7. Sablotzki A, Welters I, Lehmann N, Menges T, Gorlach G, Dehne M, et al. Plasma levels of immunoinhibitory cytokines interleukin-10 and transforming growth factor-beta in patients undergoing coronary artery bypass grafting. Eur J Cardiothorac Surg. 1997;11:763-8.

8. Tarnok A, Schneider P. Pediatric cardiac surgery with cardiopulmonary bypass: pathways contributing to transient systemic immune suppression. Shock. 2001;16 Suppl 1:24-32.

9. Franke A, Lante W, Fackeldey V, Becker HP, Thode C, Kuhlmann WD, et al. Proinflammatory and antiinflammatory cytokines after cardiac operation: different cellular sources at different times. Ann Thorac Surg. 2002;74:363-70. discussion 70-1.

10. Exner R, Tamandl D, Goetzinger P, Mittlboeck M, Fuegger R, Sautner T, et al. Perioperative GLY-GLN infusion diminishes the surgery-induced period of immunosuppression: accelerated restoration of the lipopolysaccharidestimulated tumor necrosis factor-alpha response. Ann Surg. 2003;237:110-5.

11. Whitlock RP, Devereaux PJ, Teoh KH, Lamy A, Vincent J, Pogue J, et al. Methylprednisolone in patients undergoing cardiopulmonary bypass (SIRS): a randomised, double-blind, placebo-controlled trial. Lancet. 2015;386:1243-53.

12. Basu R, Pathak S, Goyal J, Chaudhry R, Goel RB, Barwal A. Use of a novel hemoadsorption device for cytokine removal as adjuvant therapy in a patient with septic shock with multi-organ dysfunction: a case study. Indian J Crit Care Med. 2014;18:822-4.

13. Society of Thoracic Surgeons Blood Conservation Guideline Task F, Ferraris VA, Brown JR, Despotis GJ, Hammon JW, Reece TB, et al. 2011 update to the Society of Thoracic Surgeons and the Society of Cardiovascular Anesthesiologists blood conservation clinical practice guidelines. Ann Thorac Surg. 2011;91:944-82.

14. Society of Thoracic Surgeons Blood Conservation Guideline Task F, Ferraris VA, Ferraris SP, Saha SP, Hessel 2nd EA, Haan CK, et al. Perioperative blood transfusion and blood conservation in cardiac surgery: the Society of Thoracic Surgeons and The Society of Cardiovascular Anesthesiologists clinical practice guideline. Ann Thorac Surg. 2007;83(5):S27-86.

15. Kushner RF, Schoeller DA. Estimation of total body water by bioelectrical impedance analysis. Am J Clin Nutr. 1986;44:417-24. 
16. Kyle UG, Bosaeus I, De Lorenzo AD, Deurenberg P, Elia M, Gomez JM, et al. Bioelectrical impedance analysis-part l: review of principles and methods. Clin Nutr. 2004;23:1226-43.

17. Nebelsiek T, Beiras-Fernandez A, Kilger E, Mohnle P, Weis F. Routine use of corticosteroids to prevent inflammation response in cardiac surgery. Recent Pat Cardiovasc Drug Discov. 2012;7:170-4.

18. Day JR, Taylor KM. The systemic inflammatory response syndrome and cardiopulmonary bypass. Int J Surg. 2005;3:129-40.

19. Kellum JA, Venkataraman R, Powner D, Elder M, Hergenroeder G, Carter M. Feasibility study of cytokine removal by hemoadsorption in brain-dead humans. Crit Care Med. 2008:36:268-72.

20. Song M, Winchester J, Albright RL, Capponi VJ, Choquette MD, Kellum JA. Cytokine removal with a novel adsorbent polymer. Blood Purif. 2004;22:428-34.

21. Kellum JA, Song M, Venkataraman R. Hemoadsorption removes tumor necrosis factor, interleukin-6, and interleukin-10, reduces nuclear factorkappaB DNA binding, and improves short-term survival in lethal endotoxemia. Crit Care Med. 2004;32:801-5.

22. Pattnaik SK, Panda B. CytoSorb-friend or foe!! Indian J Crit Care Med. 2015;19:296.

23. Bruenger F, Kizner L, Weile J, Morshuis M, Gummert JF. First successful combination of ECMO with cytokine removal therapy in cardiogenic septic shock: a case report. Int J Artif Organs. 2015;38:113-6.

24. Vocelka CR, Jones KM, Mikhova KM, Ebisu RM, Shar A, Kellum JA, et al. Role of cytokine hemoadsorption in cardiopulmonary bypass-induced ventricular dysfunction in a porcine model. J Extra Corpor Technol. 2013;45:220-7.

25. Bernardi MH, Schmidlin D, Schiferer A, Ristl R, Neugebauer T, Hiesmayr M, et al. Impact of preoperative serum creatinine on short- and long-term mortality after cardiac surgery: a cohort study. Br J Anaesth. 2015;114:53-62.

26. Warren OJ, Smith AJ, Alexiou C, Rogers PL, Jawad N, Vincent C, et al. The inflammatory response to cardiopulmonary bypass: part 1-mechanisms of pathogenesis. J Cardiothorac Vasc Anesth. 2009;23:223-31.

27. Clark MA, Plank LD, Connolly AB, Streat SJ, Hill AA, Gupta R, et al. Effect of a chimeric antibody to tumor necrosis factor-alpha on cytokine and physiologic responses in patients with severe sepsis-a randomized, clinical trial. Crit Care Med. 1998;26:1650-9.

28. Zhang WR, Garg AX, Coca SG, Devereaux PJ, Eikelboom J, Kavsak P, et al Plasma IL-6 and IL-10 Concentrations Predict AKI and Long-Term Mortality in Adults after Cardiac Surgery. J Am Soc Nephrol. 2015;26:3123-32.

29. Frasnelli SC, de Medeiros MC, Bastos Ade S, Costa DL, Orrico SR, Rossa JC. Modulation of immune response by RAGE and TLR4 signalling in PBMCs of diabetic and non-diabetic patients. Scand J Immunol. 2015;81:66-71.

30. Tanaka Y. Immunosuppressive mechanisms in diabetes mellitus. Nippon Rinsho. 2008:66:2233-7.

31. Koh GC, Peacock SJ, van der Poll T, Wiersinga WJ. The impact of diabetes on the pathogenesis of sepsis. Eur J Clin Microbiol Infect Dis. 2012;31:379-88.

32. Wang $N$, Min X, Li D, He P, Zhao L. Geranylgeranylacetone protects against myocardial ischemia and reperfusion injury by inhibiting high-mobility group box 1 protein in rats. Mol Med Rep. 2012;5:521-4.

33. Gratia S, Kay L, Potenza L, Seffouh A, Novel-Chate V, Schnebelen C, et al. Inhibition of AMPK signalling by doxorubicin: at the crossroads of the cardiac responses to energetic, oxidative, and genotoxic stress. Cardiovasc Res. 2012;95:290-9.

34. Bangert A, Andrassy M, Muller AM, Bockstahler M, Fischer A, Volz CH, et al. Critical role of RAGE and HMGB1 in inflammatory heart disease. Proc Natl Acad Sci U S A. 2016;113:E155-64.

35. Asavarut $P$, Zhao H, Gu J, Ma D. The role of HMGB1 in inflammationmediated organ injury. Acta Anaesthesiol Taiwan. 2013;51:28-33.

36. O'Neal Jr HR, Koyama T, Koehler EA, Siew E, Curtis BR, Fremont RD, et al. Prehospital statin and aspirin use and the prevalence of severe sepsis and acute lung injury/acute respiratory distress syndrome. Crit Care Med. 2011;39:1343-50.

37. Landis RC, Brown JR, Fitzgerald D, Likosky DS, Shore-Lesserson L, Baker RA, et al. Attenuating the Systemic Inflammatory Response to Adult Cardiopulmonary Bypass: A Critical Review of the Evidence Base. J Extra Corpor Technol. 2014;46:197-211.

38. Paparella D, Micelli M, Favoino B, D'Alo M, Fiore T, de Luca Tupputi Schinosa L. Anti-heparin-platelet factor 4 antibodies after cardiopulmonary bypass: role of HLA expression. Haematologica. 2001;86:326-7.

\section{Submit your next manuscript to BioMed Central and we will help you at every step:}

- We accept pre-submission inquiries

- Our selector tool helps you to find the most relevant journal

- We provide round the clock customer support

- Convenient online submission

- Thorough peer review

- Inclusion in PubMed and all major indexing services

- Maximum visibility for your research

Submit your manuscript at www.biomedcentral.com/submit

) Biomed Central 